Revista de Matemática: Teoría y Aplicaciones 2002 9(2) : 15-30

CIMPA - UCR - CCSS ISSN: 1409-2433

\title{
UN MODELO DE EQUILIBRIO PARA ECONOMÍAS DE PRODUCCIÓN CON ANÁLISIS DE ACTIVIDAD
}

\author{
Osvaldo Acuña Ortega*
}

Recibido: 10 May 2001

\begin{abstract}
Resumen
En el presente trabajo se desarrolla un modelo para economías de producción con análisis de actividad. En este modelo se consideran los conceptos de equilibrio regular y de economías regulares. Se demuestra que éstos, se reducen a los conceptos correspondientes para el caso de economías de intercambio estudiados en [1], [8]. Se prueba también un teorema del índice para economías regulares. Todas las pruebas y argumentos matemáticos empleados en el trabajo son lo suficientemente simples para que sean entendidos por lectores sin una formación matemática sólida, de tal manera que este modelo está al alcance de estudiantes de economía avanzados. El modelo desarrollado en este artículo está inspirado en el trabajo de Kehoe [6].
\end{abstract}

Palabras clave: Economía regular, equilibrio económico, equilibrio regular.

\begin{abstract}
In this paper we study a general equilibrium model for economies with activities analysis production technologies and we prove a global index theorem for regular economies.
\end{abstract}

Keywords: Regular economy, economic equilibrium, regular equilibrium.

Mathematics Subject Classification: 90A14

\section{Introducción}

Suponemos que existen un número finito $n$ de bienes perfectamente divisibles. Tenemos una función de exceso de demanda. $f(p)=\left(f_{1}(p), \cdots, f_{n}(p)\right) \in \mathbb{R}^{n}$, definida para cada vector $p \in \mathbb{R}^{n}$ de entrada no negativas, no nulo; es decir el dominio de $f$ es $\mathbb{R}_{+}^{n} \backslash\{0\}$, donde $\mathbb{R}_{+}^{n}=\left\{p \in \mathbb{R}^{n} / p_{i} \geq s 0\right.$ para cada $\left.i=1, \cdots, n\right\}$. Todo $p \in \mathbb{R}_{+}^{n} \backslash\{0\}$ es llamado un vector de precios.

\footnotetext{
${ }^{*}$ CIMPA, Universidad de Costa Rica, 2060 San José, Costa Rica. E-Mail: oacuna@emate.ucr.ac.cr
} 
Supuesto 1. (Diferenciabilidad)

Cada $f_{i}$ es una función continuamente diferenciable en $\mathbb{R}_{+}^{n} \backslash\{0\}$.

Supuesto 2. (Homogeneidad)

Cada $f_{i}$ es homogénea de grado cero; es decir $f_{i}(t p)=f_{i}(p)$ para cada $t>0$.

Este supuesto implica que $\partial f(p) \cdot p=0$. Como $f(t p)=f(p)$; derivando está ecuación con respecto a $t$ obtenemos que $\partial f(t p) \cdot p=0$ y haciendo $t=1$ obtenemos $\partial f(p) \cdot p=0$.

Supuesto 3. (ley de Walras).

La función $f$ satisface la ecuación $p \cdot f(p)=0$ para todo $p \in \mathbb{R}_{+}^{n} \backslash\{0\}$.

La tecnología de producción está específicada por una matriz $A \quad n \times m$, tal que $A=\left[b_{1}, \cdots, b_{m}\right]$ donde $\operatorname{los} b_{i}$ son los vectores columna de $A$, los cuáles son llamados las actividades de $A$ y la matriz $A$ se llama matriz de análisis de actividad. El conjunto

$Y=\left\{A y / y \in \mathbb{R}_{+}^{m}\right\}$ es llamado el conjunto de producción. Sea $\mathbb{R}_{++}^{n}=\left\{x \in \mathbb{R}^{n} / x_{i}>0\right.$ para todo $\left.i=1, \cdots, n\right\}$.

Supuesto 4. (Desperdicio).

La matriz $-I_{n}$ es una submatriz de $A$, donde $I_{n}$ es la matriz identidad $n \times n$.

Teorema 1 Sea $A=\left[b_{1}, \cdots, b_{m}\right]$ una matriz de análisis de actividad, entonces las siguientes condiciones son equivalentes:

(i) $Y \cap \mathbb{R}_{+}^{n}=\{0\}$.

(ii) Todo elemento de $Y$ diferente de cero tiene al menos una entrada estrictamente negativa.

(iii) Existe un vector $p \in \mathbb{R}_{++}^{n}$ tal que el vector $p \cdot A$ tiene todas sus entradas no positivas, es $\operatorname{decir} p \cdot A \leq 0$.

Prueba.

(i) $\Rightarrow$ (ii). Sea $y \in \mathbb{R}_{+}^{m}$ tal que $A y \neq 0$. Si $A y$ no tiene entradas no negativas entonces $A y \in \mathbb{R}_{+}^{n}$ y por (i) $A y=0$, lo cual es una contradicción y entonces $A y$ tiene al menos una entrada estrictamente negativa.

(ii) $\Rightarrow$ (i). Sea $y \in \mathbb{R}_{+}^{m}$ tal que $A y \in \mathbb{R}_{+}^{n}$. Si $A y \neq 0$ entonces $A y$ tiene una entrada estrictamente negativa, luego $A y \notin \mathbb{R}_{+}^{n}$, esto es una contradicción; por lo tanto $A y=0$. Así tenemos que $Y \cap \mathbb{R}_{+}^{n}=\{0\}$.

(iii) $\Rightarrow$ (i). Sea $p \in \mathbb{R}_{++}^{n}$ tal que $p \cdot A \leq 0$. Si $y \in \mathbb{R}_{+}^{m}$ y $A y \in \mathbb{R}_{+}^{n}$, supongamos que $A y \neq 0$ y entonces $p \cdot(A y)>0$. Pero $p \cdot A \leq 0$ y $y \in \mathbb{R}_{+}^{m}$ implican que $(p A) \cdot y \leq 0$, lo cual es una contradicción y entonces debemos tener que $A y=0$; es decir $Y \cap \mathbb{R}_{+}^{n}=\{0\}$.

(i) $\Rightarrow$ (iii). Sea $Y^{*}=\left\{p \in \mathbb{R}^{n} / p \cdot y \leq 0\right.$ para todo $\left.y \in Y\right\}$. Si existe $p \in Y^{*}$ tal que $p \in \mathbb{R}_{++}^{n}$ entonces $p \cdot y \leq 0$ para todo $y \in Y$. Como todas las columnas de $A$ son elementos de $Y\left(b_{i}=A e_{i}, i=1, \cdots, m\right)$ donde $\left(e_{1}, \cdots, e_{m}\right)$ es la base canónica de $\mathbb{R}^{m}$ tenemos que $p \cdot b_{i} \leq 0$ para $i=1, \cdots, m$; es decir $p \cdot A \leq 0$ y entonces quedaría establecido 
(iii).

Suponga por contradicción que $\mathbb{R}_{++}^{n} \cap Y^{*}=\emptyset$; esto implica que $\mathbb{R}_{++}^{n}-Y^{*}$ no contiene el vector 0 . Como $\mathbb{R}_{++}^{n}-Y^{*}$ es un conjunto conexo $\left(\mathbb{R}_{++}^{n}, Y^{*}\right.$ son conexos), entonces por el teorema del hiperplano de soporte, existe $q \in \mathbb{R}^{n}, q \neq 0$ tal que $0 \leq q \cdot \beta$ para todo $\beta \in \mathbb{R}_{++}^{n}-Y^{*}$.

Por otro lado, como $0 \in Y^{*}$ tenemos $\mathbb{R}_{++}^{n} \subseteq \mathbb{R}_{++}^{n}-Y^{*}$ y entonces $0 \leq q \cdot x$ para todo $x \in \mathbb{R}_{++}^{n}$.

Sea $x_{i}^{e}$ el vector de $\mathbb{R}_{++}^{n}$ que tiene a $e>0$ en toda entrada $j \neq i$ y a 1 en la entrada $i$; claramente $x_{i}^{e} \rightarrow e_{i}$ si $e \rightarrow 0$ y entonces como $0 \leq q \cdot x_{i}^{e}$ se tiene $0 \leq q \cdot e_{i}$ para todo $i=1, \cdots n$ luego $q \in \mathbb{R}_{+}^{n}$. Para $e>0$ tenemos que $(e, \cdots, e)-y \in \mathbb{R}_{++}^{n}-Y^{*}$ para todo $y \in Y^{*}$ y entonces $0 \leq((e, \cdots, e)-y) \cdot q$. Cuando $e \longrightarrow 0$ se obtiene que $-y \cdot q \geq 0$; es decir $q \cdot y \leq 0$ para todo $y \in Y^{*}$.

Sea $p \in \mathbb{R}^{n}$ tal que $p \cdot b_{j} \geq 0$ para todo $j=1, \cdots, m$. Para todo $y \in Y$ existe $z \in \mathbb{R}_{+}^{m}$ tal que $y=A z$, sea $z=\left(z_{1}, \cdots, z_{m}\right)$ entonces $y=A z=\sum_{j=1}^{m} z_{j} b_{j}$. Por lo tanto se tiene que $y \cdot p=\sum_{j=1}^{m} z_{j} b_{j} p \geq 0$; es decir $-p \in Y^{*}$ y así $-p \cdot q \leq 0$ entonces $p \cdot q \geq 0$. Hemos demostrado que para todo $p \in \mathbb{R}^{n}$ tal que $p \cdot b_{j} \geq 0$ para todo $j=1, \cdots, m$ se tiene que $p \cdot q \geq 0$. Por el Lema de Minkowski-Farkas existen $\lambda_{1}, \cdots, \lambda_{m} \geq 0$ con $\left(\lambda_{1}, \cdots, \lambda_{m}\right) \neq 0$ tal que $q=\sum_{j=1}^{m} \lambda_{i} b_{i}$. Sea $z=\left(\lambda_{1}, \cdots, \lambda_{m}\right)$ entonces $q=A z$, como $z \in \mathbb{R}_{+}^{m}$ tenemos $q \in Y$; por lo tanto $q \in Y \cap \mathbb{R}_{++}^{n}=\{0\}$ así $q=0$; lo cual es una contradicción.

Supuesto 5. (Acotabilidad)

$Y \cap \mathbb{R}_{+}^{n}=\{0\}$.

Nota. Todo par de la forma $(f, A)$ es llamado una economía.

Definición 1 Un equilibrio de una economía $(f, A)$ es un vector de precios $\hat{p}$ tal que:

(i) $\hat{p}^{t} \cdot A \leq 0$.

(ii) Existe $z \in \mathbb{R}_{+}^{m}$ tal que $f(\hat{p})=A z$.

(iii) $\sum_{i=1}^{n} \hat{p}_{i}=1$.

\section{Observaciones.}

(a) Cuando una economía $(f, A)$ es tal que $A=-I_{n}$ y $f$ está definida en $\mathbb{R}_{++}^{n}$, esta se reduce al caso de economías de intercambio, estudiadas por ejemplo en [1], [8]. En este caso tenemos que $Y=-\mathbb{R}_{+}^{n}$.

(b) La noción de punto de equilibrio de la definición anterior generaliza la noción de punto de equilibrio $(f(\hat{p})=0)$ para economías de intercambio estudiada en [1], donde $f$ está definida en $\mathbb{R}_{++}^{n}$.

Probemos (b): Sea $\left(f,-I_{n}\right)$ una economía tal que el dominio de $f$ es $\mathbb{R}_{++}^{n}$ y $\hat{p} \in \mathbb{R}_{++}^{n}$ tal que $\sum_{i=1}^{n} \hat{p}_{i}=1$. Si $f(\hat{p})=0$ entonces claramente $\hat{p}^{t}\left(-I_{n}\right)=-\hat{p}^{t} \leq 0$ y $f(\hat{p})=0=\left(-I_{n}\right) z$, con $z=0 \in \mathbb{R}_{+}^{n}$. Por lo tanto $\hat{p}$ es un punto de equilibrio de acuerdo a la definición anterior. Recíprocamente si $\hat{p}$ es un punto de equilibrio de $\left(f,-I_{n}\right)$ entonces $f(\hat{p}) \in Y=-\mathbb{R}_{+}^{n}$. Si $f(\hat{p}) \neq 0$, este vector tiene una componente $f_{i}(p)<0$ y entonces $\hat{p} \cdot f(\hat{p}) \leq p_{i} f_{i}(p)<0$, contradiciendo el supuesto 3 (Ley de Walras) y por lo tanto $f(\hat{p})=0$. 
Definición 2 (a) Sea $T$ un subconjunto cerrado, convexo y no vacío de $\mathbb{R}^{n}$, denote por $p_{T}: \mathbb{R}^{n} \longrightarrow T$ la proyección definida tal que para cada $q \in \mathbb{R}^{n}, p_{T}(q)$ es el punto de $T$ más cercano a $q$, con la distancia usual de $\mathbb{R}^{n}$.

(b) Sea $S=\left\{p \in \mathbb{R}_{+}^{n} / \sum_{i=1}^{n} p_{i}=1\right\}$ y $S_{A}=\left\{p \in \mathbb{R}^{n} / p^{t} A \leq 0, \sum_{i=1}^{n} p_{i}=1\right\}$.

Es conocido que toda proyección $p_{T}$ es una función continua si $T$ es convexo.

Proposición $2 S_{A}$ es un subconjunto cerrado, conexo no vacío de $\mathbb{R}^{n}$ y $S_{A} \subseteq S$.

Prueba. Claramente $S_{A}$ es un subconjunto cerrado conexo de $\mathbb{R}^{n}$.

El teorema 1 y el supuesto 5 garantiza que existe $p \in \mathbb{R}_{++}^{n}$ tal que $p^{t} A \leq 0$; por lo tanto $\frac{p}{\sum_{i} p_{i}} \in S_{A}$ y así tenemos que $S_{A} \neq \emptyset$.

Probemos que $S_{A} \subseteq S$, sea $p \in S_{A}$ entonces $p^{t} A \leq 0$. Como $-I_{n}$ es una submatriz de $A$ (supuesto 4 ), tenemos en particular que $p^{t}\left(-I_{n}\right) \leq 0$ y entonces $p \geq 0$. Dado que $\sum_{i=1}^{n} p_{i}=1$ tenemos que $p \in S$.

Definición 3 Sea $g: S \longrightarrow S$ la función definida tal que $g(p)=p_{S_{A}}(p+f(p))$.

Nota. Como $p_{S_{A}}$ y $f$ son continuas entonces $g$ es una función continua.

Teorema 3 Sea $\hat{p} \in S$, entonces $\hat{p}$ es un punto de equilibrio de $(f, A)$ si y solo si $g(\hat{p})=\hat{p}$.

Prueba. Observe que $g(\hat{p})=\hat{p}$ si solo si $\hat{p}$ es la solución única del problema de programación cuadrática:

$$
\min \frac{1}{2}\|p-\hat{p}-f(\hat{p})\|
$$

sujeto a $p^{t} A \leq 0$ y $p^{t} e=1$, donde $e=(1, \cdots, 1)$.

Por las condiciones de Kuhn-Turker, existe $\hat{y}=\left(\hat{y}_{1}, \cdots, \hat{y}_{m}\right)$ con entradas no negativas y $\hat{\lambda} \geq 0$ tal que

$$
p-\hat{p}-f(\hat{p})+A y+\hat{\lambda} e=0 \text { у } p^{t} \cdot A \hat{y}=0 .
$$

Si $g(\hat{p})=\hat{p}$ entonces $p=\hat{p}$, lo que implica que $-f(\hat{p})+A \hat{y}+\hat{\lambda} e=0$; multiplicando esta ecuación por $\hat{p}$, tenemos que $-\hat{p} f(\hat{p})+\hat{p}^{t} A \hat{y}+\hat{\lambda}=0$; como $\hat{p}^{t} A \hat{y}=0$ y $\hat{p} f(\hat{p})=0$ entonces $\hat{\lambda}=0$. Por lo tanto $A \hat{y}=f(\hat{p})$ y como $\sum_{i=1}^{n} \hat{p}_{i}=1$ se tiene que $\hat{p}$ es un equilibrio para $(f, A)$.

Recíprocamente, si $\hat{p}$ es un punto de equilibrio para $(f, A), \hat{p}$ satisface las condiciones de Kuhn-Turker $(*)$ para el problema de programación cuadrática considerado arriba, para $\hat{\lambda}=0$ y como $\hat{p} \in S_{A}$ y $f(\hat{p})=A \hat{y}$ con $\hat{y} \in \mathbb{R}_{+}^{m}$. Entonces $\hat{p}-\hat{p}-f(\hat{p})+A \hat{y}+0 e=0$, dado que $\hat{p} f(\hat{p})=0$ se tiene $\hat{p} A \hat{y}=0$. Por lo tanto $\hat{p}$ es solución de este problema y $g(\hat{p})=\hat{p}$.

Teorema 4 Toda economía $(f, A)$ que satisface los supuestos del 1 al 5 tiene un punto de equilibrio.

Prueba. El teorema del punto fijo de Brower garantiza la existencia de un punto fijo $\hat{p}$ para $g: S \longrightarrow S$; y por el teorema $3 \hat{p}$ es un punto de equilibrio para $(f, A)$

En el resto del trabajo, estaremos interesados en las condiciones para la unicidad (al menos local) del equilibrio para una economía $(f, A)$. 
Denotaremos por $X$ una subvariedad $C^{1}$ n-dimensional con frontera de $\mathbb{R}^{n}$, que contiene en su interior a $S$ y tal que $0 \notin X$. $X$ puede ser tomada como la n-bola cerrada con centro $(a, \cdots, a)$ y radio $\sqrt{\left((p-a)^{2}+(n-1) a^{2}\right)}$ tal que $a, p$ satisfacen las desigualdades $\frac{1}{2}<a<1$ y $0<p<2 a-1$. Un simple cálculo demuestra que $e_{1}, \cdots, e_{n}$ base cánonica de $\mathbb{R}^{n}$ está en el interior de $X$ y que $(0, \cdots, 0)$ está en el exterior de $X$. Como $X$ es un conjunto convexo entonces $S$ está en el interior de $X$.

Nota. Queremos extender la función $g$ a $X$ tal que $g: X \longrightarrow S_{A} \subseteq S \subseteq X$. Para esto es suficiente extender $f$ de $S$ a $X$ y por lo tanto $g(x)=p_{S_{A}}(x+f(x))$ para todo $x \in X$.

Lema 5 Sea $f: \mathbb{R}_{+}^{n} \longrightarrow \mathbb{R}_{n}$ una función $C^{1}$. Entonces $f \mid S$ se puede extender a una función $C^{1}$ con dominio $X$.

Prueba. Es suficiente construir una función $f^{*}: \mathbb{R}^{n} \longrightarrow \mathbb{R}^{n}, C^{1}$, tal que $f^{*}(p)=f(p)$ para todo $p \in X \cap \mathbb{R}_{+}^{n}$.

La función $h: X \longrightarrow[0, \infty[$ dado por $h(x)=\|x\|$ alcanza su mínimo $\alpha$ en $X$, dado que $X$ es un conjunto compacto en $\mathbb{R}^{n}$ y como $0 \notin X$ se tiene que $\alpha>0$. Sea $\left.\beta \in\right] 0, \alpha[$, existe una función $C^{\infty}, \vartheta: \mathbb{R}^{n} \longrightarrow \mathbb{R}$ tal que $\vartheta(p)=1$ si $\|p\| \leq \beta, \vartheta(p)=0$ si $\|p\| \geq \alpha$ y $0<\vartheta(p)<1$, si $\beta<\|p\|<\alpha$. (Ver ejercicio 18, página 7 del libro [5]).

Defina $f^{o}: \mathbb{R}_{+}^{n} \longrightarrow \mathbb{R}^{n}$ tal que $f^{o}(p)=\left\{\begin{array}{ll}(1-\vartheta(p)) f(p) & \text { si } p \in \mathbb{R}_{+}^{n} \backslash\{0\} \\ 0 & \text { si } p=0\end{array}\right.$ Entonces si $p \in \mathbb{R}_{+}^{n} \cap X$ implica que $\|p\| \geq \alpha$ y entonces $\vartheta(p)=0$ y $f^{o}(p)=f(p)$.

Note que $f^{o}$ es $C^{1}$ ya que $f^{o} \mid \mathbb{R}_{+}^{n} \backslash\{0\}=(1-\theta) \cdot f$ y como $f^{0} \mid B(0, \beta) \cap \mathbb{R}_{+}^{n} \equiv 0, f^{0}$ es $C^{1}$ en 0 . Por lo tanto $f^{o}$ es $C^{1}$ en $\mathbb{R}_{+}^{n}$.

El lema quedaría probado cuando extendamos $f^{o}$ a una función $f^{*}: \mathbb{R}^{n} \longrightarrow \mathbb{R}^{n}, C^{1}$.

Defina $f^{i}:\left\{p \in \mathbb{R}^{n} / p_{i+1} \geq 0, \cdots, p_{n} \geq 0\right\} \longrightarrow \mathbb{R}^{n}, C^{1}$, para $i=0,1, \cdots, n$ por inducción sobre $i$. $f^{o}$ se definió arriba y suponga que $f^{i-1}$ está definida y es $C^{1}$. Definimos $f^{i}:\left\{p \in \mathbb{R}^{n} / p_{i+1} \geq 0, \cdots, p_{n} \geq 0\right\} \longrightarrow \mathbb{R}^{n}$ tal que

$$
f^{i}\left(p_{1}, \cdots, p_{n}\right)=\left\{\begin{array}{lr}
f^{i-1}\left(p_{1}, \cdots, p_{n}\right) & \text { si } p_{i} \geq 0 \\
-f^{i-1}\left(p_{1}, \cdots,-p_{i}, p_{i+1}, \cdots, p_{n}\right) & \text { si } p_{i}<0 \\
+2 f^{i-1}\left(p_{1}, \cdots, p_{i-1}, 0, p_{i+1}, \cdots, p_{n}\right) &
\end{array}\right.
$$

Claramente $f^{i}$ es una extensión de $f^{i-1}$. Probemos que $f^{i}$ es $C^{1}$. Sea $j \in\{1, \cdots, n\}$ y $p$ en el dominio $f^{i}$. Si $p_{i}>0$ tenemos que $\frac{\partial f^{i}}{\partial x_{j}}(p)$ existe y $\frac{\partial f^{i}}{\partial x_{j}}(p)=\frac{\partial f^{i-1}}{\partial x_{j}}(p)$ ya que $f^{i}=f^{i-1}$ en

$$
\left\{x \in \mathbb{R}^{n} / x_{i}>0, x_{i+1} \geq 0, \cdots, x_{n} \geq 0\right\} .
$$

Si $p_{i}<0$ y $i \neq j$, como en el caso anterior tenemos:

$$
\frac{\partial f^{i}}{\partial x_{j}}(p)=\frac{\partial f^{i-1}}{\partial x_{j}}\left(p_{1}, \cdots, p_{i-1},-p_{i}, p_{i+1}, \cdots, p_{n}\right)+2 \frac{\partial f^{i-1}}{\partial x_{j}}\left(p_{1}, \cdots, p_{i-1}, 0, p_{i+1}, \cdots, p_{n}\right) .
$$

Si $p_{i}<0$ y $i=j$ aplicando la regla de la cadena tenemos

$$
\frac{\partial f^{i}}{\partial x_{i}}(p)=\frac{\partial f^{i-1}}{\partial x_{i}}\left(p_{1}, \cdots, p_{i-1},-p_{i}, p_{i+1}, \cdots, p_{n}\right)
$$


Si $p_{i}=0$ considere el límite:

$$
\begin{aligned}
\lim _{\substack{h \rightarrow 0 \\
h>0}} & \frac{f^{i}(p+h e i)-f^{i}(p)}{h} \\
\quad= & \lim _{\substack{h \rightarrow 0 \\
h>0}} \frac{f^{i-1}\left(p_{1}, \cdots, p_{i-1}, h, p_{i+1}, \cdots, p_{n}\right)-f^{i-1}\left(p_{1}, \cdots, p_{i-1}, 0, p_{i+1}, \cdots, p_{n}\right)}{h} \\
= & \frac{\partial f^{i-1}}{\partial x_{i}}\left(p_{1}, \cdots, p_{i-1}, 0, p_{i+1}, \cdots, p_{n}\right)=\frac{\partial f^{i-1}}{\partial x_{i}}(p) .
\end{aligned}
$$

Por otro lado considere

$$
\begin{aligned}
\lim _{\substack{h \rightarrow 0 \\
h<0}} \frac{f^{i}(p+h e i)-f^{i}(p)}{h} \\
=\lim _{\substack{h \rightarrow 0 \\
h<0}} \frac{-f^{i-1}\left(p_{1}, \cdots, p_{i-1},-h, p_{i+1}, \cdots, p_{n}\right)+2 f^{i-1}\left(p_{1}, \cdots, p_{i-1}, 0, p_{i+1}, \cdots, p_{n}\right)-f^{i-1}(p)}{h} \\
=\lim _{\substack{h \rightarrow 0 \\
h<0}} \frac{-f^{i-1}\left(p_{1}, \cdots, p_{i-1},-h, p_{i+1}, \cdots, p_{n}\right)+f^{i-1}(p)}{h} \\
=\lim _{\substack{h \rightarrow 0 \\
h<0}} \frac{f^{i-1}\left(p_{1}, \cdots, p_{i-1},-h, p_{i+1}, \cdots, p_{n}\right)-f^{i-1}(p)}{-h} \\
=\frac{\partial f^{i-1}}{\partial x_{i}}(p) .
\end{aligned}
$$

Por lo tanto $\frac{\partial f^{i}}{\partial x_{i}}(p)$ existe si $p_{i}=0$ y $\frac{\partial f^{i}}{\partial x_{i}}(p)=\frac{\partial f^{i-1}}{\partial x_{i}}(p)$.

Si $i \neq j$ y $p_{i}=0$ considere el límite

$$
\lim _{h \rightarrow 0} \frac{f^{i}(p+h e j)-f^{i}(p)}{h}=\lim _{h \rightarrow 0} \frac{f^{i-1}(p+h e j)-f^{i-1}(p)}{h}=\frac{\partial f^{i-1}}{\partial x_{j}}(p) .
$$

Por lo tanto $\frac{\partial f^{i}}{\partial x_{j}}(p)=\frac{\partial f^{i-1}}{\partial x_{j}}(p)$ cuando $p_{i}=0$ y $i \neq j$.

Hemos probado que:

$$
\frac{\partial f^{i}}{\partial x_{j}}\left(p_{1}, \cdots, p_{n}\right)= \begin{cases}\frac{\partial f^{i-1}}{\partial x_{j}}\left(p_{1}, \cdots, p_{n}\right) & \text { si } p_{i} \geq 0 \\ -\frac{\partial f^{i-1}}{\partial x_{j}}\left(p_{1}, \cdots, p_{i-1},-p_{i}, p_{i+1}, \cdots, p_{n}\right) & \text { si } p_{i}<0 \\ +2 \frac{\partial f^{i-1}}{\partial x_{j}}\left(p_{1}, \cdots, p_{i-1}, 0, p_{i+1}, \cdots, p_{n}\right) & \end{cases}
$$

cuando $i \neq j$, y

$$
\frac{\partial f^{i}}{\partial x_{j}}\left(p_{1}, \cdots, p_{n}\right)= \begin{cases}\frac{\partial f^{i-1}}{\partial x_{i}}\left(p_{1}, \cdots, p_{n}\right) & \text { si } p_{i} \geq 0 \\ \frac{\partial f^{i-1}}{\partial x_{i}}\left(p_{1}, \cdots, p_{n}\right) & \text { si } p_{i}<0\end{cases}
$$

cuando $i=j$.

Luego $\frac{\partial f^{i}}{\partial x_{j}}$ es continua dado que $\frac{\partial f^{i-1}}{\partial x_{j}}$ es continua; y así tenemos que $f^{i}$ es $C^{1}$, completándose la inducción. Sea $f^{*}=f^{n}$, como $f^{n}$ es una extensión de $f^{o}$ y $f^{n}$ está definida en $\mathbb{R}^{n}$, el lema queda probado. 
Nota. El conjunto de puntos fijos de $g: X \longrightarrow X$ es igual al conjunto de puntos fijos de $g \mid S: S \longrightarrow S$, ya que como $g(X) \subseteq S_{A} \subseteq S$, entonces si $g(p)=p$ se tiene que $p \in S_{A}$ y luego $p \in S$.

Supuesto 6 . Ninguna columna de $A$ se puede expresar como combinación lineal de otras columnas de $A$ que sean menos de $n$.

Este supuesto es trivialmente satisfecho si $A=-I_{n}$ ya que en este caso las columnas de $A$ son una base de $\mathbb{R}^{n}$.

Definición 4 Sea $p \in \mathbb{R}_{+}^{n} \backslash\{0\}$ y $B(p)$ la submatriz de $A$ obtenida suprimiendo todas las columnas $b_{i}$ de $A$ tales que $p b_{i} \neq 0$.

Cuando $A=-I_{n}$ y $p \in \mathbb{R}_{++}^{n}$, $B(p)$ es la matriz vacía ( no tiene entradas ).

Nota. Sea $(f, A)$, una economía con $A=\left[b_{1}, \cdots, b_{m}\right]$ matriz definida por columnas. Si $\hat{p}$ es un equilibrio para $(f, A)$ existe $\hat{y} \in \mathbb{R}_{+}^{m}$ tal que

$$
\begin{aligned}
f(\hat{p}) & =A \hat{y}=\left[b_{1}, \cdots, b_{m}\right] \cdot \hat{y} \\
& =\left[\begin{array}{ccc}
b_{11}, & \cdots, & b_{m 1} \\
& \\
b_{1 n}, & \cdots, & b_{m n}
\end{array}\right] \cdot\left[\begin{array}{c}
\hat{y}_{1} \\
\vdots \\
\hat{y}_{m}
\end{array}\right]=\left[\begin{array}{c}
\sum_{j=1}^{m} b_{j 1} \hat{y}_{j} \\
\vdots \\
\sum_{j=1}^{m} b_{j m} \hat{y}_{j}
\end{array}\right]=\sum_{j=1}^{m} \hat{y}_{j} b_{j} .
\end{aligned}
$$

Por lo tanto $f(\hat{p})=\sum_{j=1}^{m} \hat{y}_{j} b_{j}=\sum_{b_{j} \in B(\hat{p})} \hat{y}_{j} b_{j}+\sum_{b_{j} \notin B(\hat{p})} \hat{y}_{j} b_{j} .\left(b_{j} \in B(\hat{p})\right.$ significa que $b_{j}$ es una columna de $B(\hat{p})$ y $b_{j} \notin B(\hat{p})$ significa que $b_{j}$ es una columna de $A$ que no es una columna de $B(\hat{p})$.)

Como $\hat{p} \cdot A \leq 0$ entonces $\hat{p} \cdot b_{j}<0$ para todo $b_{j} \notin B(\hat{p})$ y $\hat{p} \cdot b_{j}=0$ para todo $b_{j} \in B(\hat{p})$ y entonces se tiene que: $0=\hat{p} \cdot f(\hat{p})=\sum_{b_{j} \notin B(\hat{p})} \hat{y}_{j} \hat{p} \cdot b_{j}$; como $\hat{p} \cdot b_{j}<0$ si $b_{j} \notin B(\hat{p})$ y dado que $\hat{y}_{j} \geq 0$ para todo $j$, tenemos que $\hat{y}_{j}=0$.

si $b_{j} \notin B(\hat{p})$ y entonces $f(\hat{p})=\sum_{b_{j} \in B(\hat{p})} \hat{y}_{j} b_{j}$.

En particular si $B(\hat{p})$ es la matriz vacía entonces $f(\hat{p})=0$.

Supuesto 7. En cada punto de equilibrio $\hat{p}$ de $(f, A)$ tenemos que $\hat{y}_{j}>0$ para cada $j$ tal que $b_{j} \cdot \hat{p}=0$, siendo $b_{j}$ una columna de $A$.

Nota. Si $A=-I_{n}$ este supuesto es trivialmente satisfecho ya que $B(\hat{p})$ es vacia.

Proposición 6 Para $p \in \mathbb{R}_{+}^{n} \backslash\{0\}$, tenemos que las columnas de $B(p)$ son linealmente independientes y son estrictamente menos de $n$.

Prueba. Si $B(p)$ es vacía la proposición es trivial. Suponga que $B(p)$ no es la matriz vacía y sean $b_{i_{1}}, \cdots, b_{i_{l}}$ las columnas de $B(p)$. Escoja $\left\{b_{j_{1}}, \cdots, b_{j_{r}}\right\} \subseteq\left\{b_{i_{1}}, \cdots, b_{i_{l}}\right\}$ subconjunto linealmente independiente tal que $<b_{j_{1}}, \cdots, b_{j_{r}}>=<b_{i_{1}}, \cdots, b_{i_{l}}>$. Si las columnas de $B(p)$ son linealmente dependientes, existe una columna de $B(p)$ no en $b_{j_{1}}, \cdots, b_{j_{r}}$ que es combinación lineal de $b_{j_{1}}, \cdots, b_{j_{r}}$. Por el supuesto 6 tenemos que $\left|\left\{b_{j_{1}}, \cdots, b_{j_{r}}\right\}\right| \geq n$ y por lo tanto $r=n$.

Por otro lado como $p \cdot b_{j_{k}}=0$ para todo $k=1, \cdots, r$ entonces $\left\{b_{j_{1}}, \cdots, b_{j_{r}}, p\right\}$, es linealmente independiente y así tenemos que $r<n$, pero esto contradice $r=n$, luego las columnas de $B(p)$ son linealmente independientes. Como $p \cdot b_{i_{k}}=0$ para $k=1, \cdots, l$ se tiene que $\left\{b_{i_{1}}, \cdots, b_{i_{l}}, p\right\}$, es linealmente independiente y entonces $l+1 \leq n$ así $l<n$. 
Definición 5 Si $p \in \mathbb{R}_{+}^{n} \backslash\{0\}$ sea $C_{p}=\left[e, b_{i_{1}}, \cdots, b_{i_{l}}\right]$ la matriz definida por columnas, donde $B(p)=\left[b_{i_{1}}, \cdots, b_{i_{l}}\right]$ y e es el vector con todas sus entradas iguales a 1 .

Corolario 7 Las columnas de $C_{p}$ son linealmente independientes.

Prueba. Es suficiente probar que $e$ no es una combinación lineal de las columnas de $B(p)$. Como $p \in \mathbb{R}_{+}^{n} \backslash\{0\}$ se tiene que $p \cdot e>0$. Suponga por contradicción que existen $\alpha_{1}, \cdots, \alpha_{l}$ escalares tales que $e=\alpha_{1} b_{i_{1}}+\cdots+\alpha_{l} b_{i_{l}}$, como $p \cdot b_{i_{k}}=0$ para $k=1, \cdots, l$, se tiene que $p \cdot e=\alpha_{1} \cdot 0+\cdots+\alpha_{l} \cdot 0=0$, pero esto contradice $p \cdot e>0$. Luego las columnas de $C_{p}$ son linealmente independientes.

Nota. Observe que $\left\{x \in \mathbb{R}^{n} / x \cdot C_{p}=\left[\begin{array}{c}1 \\ 0 \\ \vdots \\ 0\end{array}\right]\right\}=\left\{x \in \mathbb{R}^{n} / x \cdot e=1, x \cdot B(p)=0\right\}$.

Proposición 8 Sea $C$ una matriz real con $n$ filas y $k$ columnas. Si $S=\left\{x \in \mathbb{R}^{n} / x \cdot C=\hat{c}\right\}$ es un subconjunto no vacío de $\mathbb{R}^{n}$ y las columnas de $C$ son linealmente independientes. Entonces $p_{s}$ es diferenciable y su matriz jacobiana está dada por $D_{q} p_{s}=I-C\left(C^{t} C\right)^{-1} C^{t}$, para todo $q \in \mathbb{R}^{n}$.

Prueba. Sea $C=\left[b_{1}, \cdots, b_{k}\right]$, donde $\operatorname{los} b_{i}$ son las columnas de $C$. Calculemos la función $p_{s}: \mathbb{R}^{n} \longrightarrow S$. Si $q \in \mathbb{R}^{n}$ tenemos que $p_{s}(q)$ es el punto de $s$ más cercano a $q$, entonces $y=p_{s}(q)$ resuelve el problema de minimizar la función $f(y)=\frac{1}{2}(\|y-q\|)^{2}$ sujeta a $y \cdot C=\hat{c}$.

Por lo tanto $y=p_{s}(q)$ satisface las condiciones de primer orden; es decir existe $\lambda=\left(\lambda_{1}, \cdots, \lambda_{k}\right)$ tal que:

$$
\nabla f(y)=\lambda_{1} \nabla\left(y \cdot b_{1}-\hat{c}_{1}\right)+\cdots+\lambda_{k} \nabla\left(y \cdot b_{k}-\hat{c}_{k}\right), \text { donde } \hat{c}=\left(\hat{c}_{1}, \cdots, \hat{c}_{k}\right)
$$

Como $\nabla f(y)=\left[y_{1}-q_{1}, \cdots, y_{n}-q_{n}\right]=y-q$ y $\nabla\left(y \cdot b_{i}-\hat{c}_{i}\right)=b_{i}$, para $i=1, \cdots, k$.

Entonces $y-q=\sum_{i=1}^{k} \lambda_{i} b_{i}=\lambda C^{t}$ por lo tanto $\hat{c}-q C=\lambda\left(C^{t} C\right)$.

Como las columnas de $C$ son linealmente independientes se tiene que $C^{t} C$ es invertible, y así tenemos que $\lambda=\hat{c}\left(C^{t} C\right)^{-1}-q C\left(C^{t} C\right)^{-1}$ y entonces

$$
p_{s}(q)=q+\left[\hat{c}\left(C^{t} C\right)^{-1}-q C\left(C^{t} C\right)^{-1}\right] C^{t}=q-q C\left(C^{t} C\right)^{-1} C^{t}+\hat{c}\left(C^{t} C\right)^{-1} C^{t}
$$

luego $p_{s}$ es diferenciable y $D_{q} p_{s}=I_{n}-C\left(C^{t} C\right)^{-1} C^{t}$.

Teorema 9 Sea $(f, A)$ una economía que satisface los supuestos del 1 al 7 . Entonces la función $g$ es diferenciable en algún vecindario abierto de cada punto fijo $\hat{p}$ de $g$ y tenemos que $D_{p}(g)-I_{n}=\left(I_{n}-C_{\hat{p}}\left(C_{\hat{p}}^{t} C_{\hat{p}}\right)^{-1} C_{\hat{p}}^{t}\right)\left(I_{n}+D_{p} f\right)-I_{n}$ donde $D_{p}(g)$ y $D_{p}(f)$ son las matrices jacobianas de $g$ y $f$ en $p$, punto arbitrario del vecindario de $\hat{p}$.

Prueba. Tenemos que $g: X \longrightarrow S_{A} \subseteq S \subseteq X$. Por lo tanto para todo $p \in X, g(p) \cdot e=1$ y $g(p) \cdot A \leq 0$.

Sabemos que $g(p)$ es la solución única al problema de programación cuadrática: mín $\mid(\|x-p-f(p)\|)^{2}$ sujeto a $x \cdot A \leq 0$ y $x \cdot e=1$. Por las condiciones de Kuhn-Tucker, existe $y=\left(y_{1}, \cdots, y_{m}\right)$ con entradas no negativas y $\lambda \geq 0$ tal que $g(p)-p-f(p)+A y+\lambda e=0$ 
y $g(p) \cdot A y=0$, entonces $g(p) \cdot g(p)-g(p) \cdot p-g(p) \cdot f(p)+\lambda=0$; es $\operatorname{decir} \lambda=\lambda(p)$ es una función continua en $p$, para $p \in X$.

Por otro lado sea $\hat{p}$ un punto fijo de $g$. Si $b_{j}$ es una columna de $A$ pero no de $B(\hat{p})$, tenemos que $b_{j} \cdot \hat{p}<0$ y entonces $g(\hat{p}) \cdot b_{j}<0$. Por continuidad de $g$ existe un vecindario $U$ de $\hat{p}$ tal que $g(p) \cdot b_{j}<0$ para toda $b_{j}$ columna de $A$ que no es columna de $B(\hat{p})$ y $p \in U$.

Por otro lado si $p \in U$ tenemos que: $0=g(p) A y=\sum_{j=1}^{m} y_{j} g(p) b_{j} \leq y_{i} g(p) b_{i}$ para todo $i$, ya que para $j, g(p) b_{j} \leq 0, y_{j} \geq 0$.

Por lo tanto para todo $i, y_{i} g(p) b_{i}=0$.

Si $b_{k} \notin B(\hat{p})$ se tiene $g(p) b_{k}<0$, lo que implica que $y_{k}=0$.

Por lo tanto $A y=\sum_{b_{j} \in B(\hat{p})} y_{j} b_{j}$ y entonces se tiene que

$$
\sum_{b_{j} \in B(\hat{p})} y_{j} b_{j}=-(g(p)-p-f(p)+\lambda(p) \cdot e) .
$$

Sea $V$ es el subespacio vectorial de $\mathbb{R}^{n}$ generado por las columnas de $B(\hat{p})$.

Sean $p_{V}: \mathbb{R}^{n} \longrightarrow V \mathrm{y} \Pi_{k}: V \longrightarrow \mathbb{R}$ tales que $\Pi_{k}\left(\sum_{b_{j} \in B(\hat{p})} \alpha_{j} b_{j}\right)=\alpha_{k}$, para $k=1, \cdots, \operatorname{dim} V$.

Proyecciones lineales. Estas funciones son continuas y tenemos que

$$
y_{j}=-\Pi_{j}\left(p_{V}(g(p)-p-f(p)+\lambda(p) \cdot e)\right)
$$

tal que $b_{j}$ es una columna de $B(\hat{p})$; por lo tanto $y_{j}=y_{j}(p)$ es una función continua en $p$, para $p \in U$.

Por el supuesto 7 tenemos que $y_{j}(\hat{p})=\hat{y}>0$ y entonces existe un vecindario $W$ de $\hat{p}$ tal que $W \subseteq U$ y $y_{j}(p)>0$ para todo $p \in W$ y para todo $j$ tal que $b_{j}$ es una columna de $B(\hat{p})$.

Para $p \in W$ tenemos que:

$$
0=\sum_{b_{j} \in B(\hat{p})} y_{j} g(p) b_{j}, \operatorname{con} g(p) \cdot b_{j} \leq 0 \text { y } y_{j}>0
$$

lo cual implica que $g(p) \cdot b_{j}=0$ para $j$ tal que $b_{j} \in B(\hat{p})$; es $\operatorname{decir} g(p) \cdot B(\hat{p})=0$.

Queremos probar que $g\left|W=g_{1}\right| W: W \longrightarrow S$ donde $S=\left\{x \in \mathbb{R}^{n} / x C_{\hat{p}}=\left[\begin{array}{c}1 \\ 0 \\ \vdots \\ 0\end{array}\right]\right\}$ y $g_{1}(p)=p_{s}(p+f(p))$.

Esto implicaría que $g$ es diferenciable en $W$, dado que $p_{s}$ es diferenciable (proposición 8) y $f$ es diferenciable. Por la regla de la cadena y la proposición 8 tendríamos que $D_{p} g_{1}=\left(I_{n}-C_{\hat{p}}\left(C_{\hat{p}}^{t} C_{\hat{p}}\right)^{-1} C_{\hat{p}}^{t}\right)\left(I_{n}+D_{p} f\right)$ y como $D_{p} g=D_{p} g_{1}$, implicaría $D_{p} g=\left(I_{n}-\right.$ $\left.C_{\hat{p}}\left(C_{\hat{p}}^{t} C_{\hat{p}}\right)^{-1} C_{\hat{p}}^{t}\right)\left(I_{n}+D_{p} f\right)$ y entonces $D_{p} g-I_{n}=\left(I_{n}-C_{\hat{p}}\left(C_{\hat{p}}^{t} C_{\hat{p}}\right)^{-1} C_{\hat{p}}^{t}\right)\left(I_{n}+D_{p} f\right)-I_{n}$. Sea $p \in W, g_{1}(p)$ es la solución única del problema

$$
\min \frac{1}{2}(\|x-p-f(p)\|)^{2}
$$

sujeto a $x \cdot e=1$ y $x \cdot B(\hat{p})=0$.

Si $T=\left\{x \in \mathbb{R}^{n} / x \cdot b_{j}<0\right.$ para toda $b_{j}$ columna de $A$ pero no $\left.B(\hat{p})\right\}$, entonces $T$ es un 
conjunto abierto de $\mathbb{R}^{n}$ y como $p \in W$ tenemos que $g(p) \in T$ y $g(p) \cdot B(\hat{p})=0$. Si $x \in T$ y $x \cdot B(\hat{p})=0$ como $x \cdot b_{j}<0$ para toda $b_{j} \notin B(\hat{p})$ entonces $x \cdot A \leq 0$.

Por lo tanto que $\left\{x \in \mathbb{R}^{n} / x \cdot B(\hat{p})=0, x \cdot e=1\right\} \cap T \subseteq\left\{x \in \mathbb{R}^{n} / x \cdot A \leq 0, x \cdot e=1\right\} \cap T$. Como $g(p)$ es solución del problema mín $\frac{1}{2}(\|x-p-f(p)\|)^{2}$ sujeto a $x \cdot A \leq 0, x \cdot e=1$, $g(p)$ es solución (local) del mismo problema en $x \in T, x \cdot A \leq 0, x \cdot e=1$ y entonces $g(p)$ es solución (local) del problema $\left({ }^{*}\right)^{\prime}$ en $x \cdot B(\hat{p})=0, x \cdot e=1, x \in T$.

Pero toda solución local es una solución global del problema $\left(^{*}\right)^{\prime}$, dado $\frac{1}{2}(\|x-p-f(p)\|)^{2}$ es una función convexa, y así tenemos que $g(p)$ resuelve el problema (*)'. Como esta solución es única debemos tener que $g(p)=g_{1}(p)$ para todo $p \in W$; es decir $g\left|W=g_{1}\right| W$ y así queda demostrado el teorema 9.

Nota. Si $x \in \mathbb{R}$, sea $\operatorname{sig}(x)=\frac{|x|}{x}$.

Proposición 10 Sea $C$ una matriz real $n \times k, k \leq n$, con sus $k$ columnas linealmente independientes. Si $B$ es una matriz real $n \times n$ entonces

$$
\operatorname{det}\left(\left(I_{n}-C\left(C^{t} C\right)^{-1} C^{t}\right)\left(I_{n}+B\right)-I_{n}\right)=\frac{1}{\operatorname{det}\left(C^{t} C\right)} \cdot \operatorname{det}\left[\begin{array}{ll}
B & C \\
C^{t} & 0
\end{array}\right] .
$$

En particular tenemos que

$$
\operatorname{sig}\left(\operatorname{det}\left(\left(I_{n}-C\left(C^{t} C\right)^{-1} C^{t}\right)\left(I_{n}+B\right)-I_{n}\right)\right)=\operatorname{sig}\left(\operatorname{det}\left[\begin{array}{ll}
B & C \\
C^{t} & 0
\end{array}\right]\right) .
$$

Prueba. Como las columnas de $C$ son linealmente independiente tenemos que $\operatorname{det}\left(C^{t} C\right)>0$, luego $\left(C^{t} C\right)^{-1}$ existe y la segunda ecuación sigue inmediatamente de la primera. Tenemos claramente que $\left(I_{n}-C\left(C^{t} C\right)^{-1} C^{t}\right)\left(I_{n}+B\right)-I_{n}=B-C\left(C^{t} C\right)^{-1}\left(C^{t} B+\right.$ $\left.C^{t}\right)$.

Por otro lado sabemos de propiedades básicas de determinantes que

$$
\begin{aligned}
\operatorname{det}\left[\begin{array}{ll}
B & C\left(C^{t} C\right)^{-1} \\
C^{t} B+C^{t} & I_{k}
\end{array}\right] & =\operatorname{det}\left(B-C\left(C^{t} C\right)^{-1} I_{k}^{-1}\left(C^{t} B+C^{t}\right)\right) \operatorname{det}\left(I_{k}\right) \\
& =\operatorname{det}\left(B-C\left(C^{t} C\right)^{-1}\left(C^{t} B+C^{t}\right)\right)
\end{aligned}
$$

Se tiene que $\operatorname{det}\left[\begin{array}{ll}B & C\left(C^{t} C\right)^{-1} \\ C^{t} B+C^{t} & I_{k}\end{array}\right]=\operatorname{det}\left[\begin{array}{ll}B & C\left(C^{t} C\right)^{-1} \\ C^{t} & 0\end{array}\right]$ restando la primera fila multiplicada a la derecha por $C^{t}$ a la segunda fila de la matriz en bloque izquierda. De la identidad

$$
\left[\begin{array}{ll}
B & C\left(C^{t} C\right)^{-1} \\
C^{t} & 0
\end{array}\right] \cdot\left[\begin{array}{ll}
I_{n} & 0 \\
0 & C^{t} C
\end{array}\right]=\left[\begin{array}{ll}
B & C \\
C^{t} & 0
\end{array}\right]
$$

se sigue que $\operatorname{det}\left[\begin{array}{ll}B & C\left(C^{t} C\right)^{-1} \\ C^{t} & 0\end{array}\right] \cdot \operatorname{det}\left(C^{t} C\right)=\operatorname{det}\left[\begin{array}{ll}B & C \\ C^{t} & 0\end{array}\right]$.

$$
\text { y entonces } \operatorname{det}\left(\left(I_{n}-C\left(C^{t} C\right)^{-1} C^{t}\right)\left(I_{n}+B\right)-I_{n}\right)=\frac{1}{\operatorname{det}\left(C^{t} C\right)} \cdot \operatorname{det}\left[\begin{array}{ll}
B & C \\
C^{t} & 0
\end{array}\right] \text {. }
$$

Proposición 11 Sea $(f, A)$ una economía que satisface los supuestos del 1 al 7 y sea $\hat{p}$ un punto de equilibrio de $(f, A)$ entonces $\operatorname{det}\left(D_{\hat{p}} g-I_{n}\right)=\frac{1}{\operatorname{det}\left(C^{t} C\right)} \cdot \operatorname{det}\left[\begin{array}{ll}D_{\hat{p}} f & C_{\hat{p}} \\ C_{\hat{p}}^{t} & 0\end{array}\right]$ en particular

$$
\operatorname{sig}\left(\operatorname{det}\left(D_{\hat{p}} g-I_{n}\right)\right)=\operatorname{sig}\left(\operatorname{det}\left[\begin{array}{ll}
D_{\hat{p}} f & C_{\hat{p}} \\
C_{\hat{p}}^{t} & 0
\end{array}\right]\right) .
$$


Prueba. Del teorema 9 sabemos que $D_{\hat{p}} g-I_{n}=\left(I_{n}-C_{\hat{p}}\left(C_{\hat{p}}^{t} C_{\hat{p}}\right)^{-1}\right) \cdot\left(I_{n}+D_{\hat{p}} f\right)-I_{n}$.

Por la proposición anterior tomando $B=D_{\hat{p}} f$ tenemos que: $\operatorname{det}\left(D_{\hat{p}} g-I_{n}\right)=\frac{1}{\operatorname{det}\left(C^{t} C\right)}$. $\operatorname{det}\left[\begin{array}{ll}D_{\hat{p}} f & C_{\hat{p}} \\ C_{\hat{p}}^{t} & 0\end{array}\right]$

Por el corolario $7 C_{\hat{p}}$ tiene las columnas linealmente independientes, entonces sigue inmediatamente la segunda ecuación.

Definición 6 Sea $(f, A)$ una economía que satisface los supuestos del 1 al 7 y sea $\hat{p}$ un punto de equilibrio de $(f, A)$. Si la matriz $\left[\begin{array}{ll}D_{\hat{p}} f & C_{\hat{p}} \\ C_{\hat{p}}^{t} & 0\end{array}\right]$ es no singular, decimos que $\hat{p}$ es un punto de equilibrio regular. En caso contrario decimos $\hat{p}$ es un punto de equilibrio crítico. Si todos los puntos de equilibrio de $(f, A)$ son regulares decimos que $(f, A)$ es una economía regular. Una economía que no es regular es llamada una economía crítica.

Corolario 12 Sea $(f, A)$ una economía que satisface los supuestos del 1 al 7 y sea $\hat{p}$ un punto de equilibrio.Entonces $\hat{p}$ es regular si solo si $D_{\hat{p}} g-I_{n}$ es una matriz no singular.

Nota. Sabemos que los supuestos $4,5,6,7$ son trivialmente satisfechos si $A=-I_{n}$.

Proposición 13 Sea una economía $\left(\xi,-I_{n}\right)$ de intercambio que satisface los puntos 1,2 y 3. Si $\hat{p}$ es un punto de equilibrio tal que $\hat{p}_{i}>0$ para $i=1, \cdots, n$. Entonces las siguientes condiciones son equivalentes:

(i) $\hat{p}$ es un punto de equilibrio regular.

(ii) $\left[\begin{array}{cc}D_{\hat{p}} f & e \\ e^{t} & 0\end{array}\right]$ es no singular.

(iii) $\left[\begin{array}{ll}D_{\hat{p}} f & \hat{p} \\ \hat{p}^{t} & 0\end{array}\right]$ es no singular.

(iv) $D_{\hat{p}} f$ tiene rango $n-1$.

Prueba. (i) $\Rightarrow$ (ii) sigue de la definición de punto regular y del hecho de que $C_{\hat{p}}=e$ dado que $B(\hat{p})$ es vacía. (i) $\Rightarrow$ (iv) Sabemos del teorema 9 aplicado al caso de intercambio que:

$$
\begin{aligned}
D_{\hat{p}} g & =\left(I_{n}-C_{\hat{p}}\left(C_{p}^{t} C_{p}\right)^{-1} C_{\hat{p}}^{t}\right) \cdot\left(I_{n}+D_{\hat{p}} f\right) \\
& =\left(I_{n}-e \cdot[1]^{-1} e^{t}\right) \cdot\left(I_{n}+D_{\hat{p}} f\right) \\
& =\left(I_{n}-e \cdot e^{t}\right) \cdot\left(I_{n}+D_{\hat{p}} f\right) \\
& =\left(I_{n}-E\right) \cdot\left(I_{n}+D_{\hat{p}} f\right) \\
& =-E+\left(I_{n}-E\right) \cdot D_{\hat{p}} f+I_{n}
\end{aligned}
$$

entonces $D_{\hat{p}} g-I_{n}=-E+\left(I_{n}-E\right) \cdot D_{\hat{p}} f$ donde $E$ es la matriz $n \times n$ con todas sus entradas iguales a 1 . Sea $v \in \operatorname{ker}\left(D_{\hat{p}} f\right)\left(\right.$ núcleo de $\left(D_{\hat{p}} f\right)$ ), entonces si $v \neq 0$, como $D_{\hat{p}} g-I_{n}$ es invertible (Corolario 12) se tiene $\left(D_{\hat{p}} g-I_{n}\right) v \neq 0$, luego $E(v) \neq 0$ es decir $\sum_{i=1}^{n} v_{i} \neq 0$. 
Sea $V=\left\{v \in \mathbb{R}^{n} / \sum_{i=1}^{n} v_{i}=0\right\}, V$ es un subespacio vectorial de $\mathbb{R}^{n}$ de dimensión $n-1$. El argumento de arriba prueba que $V \cap \operatorname{ker}\left(D_{\hat{p}} f\right)=\{0\}$ y entonces $n \geq \operatorname{dim}\left(V+\operatorname{ker}\left(D_{\hat{p}} f\right)\right)=$ $\operatorname{dim} V+\operatorname{dim}\left(\operatorname{ker}\left(D_{\hat{p}} f\right)\right) \geq \operatorname{dim} V+1 \geq n-1+1=n .\left(\operatorname{dim} \operatorname{ker}\left(D_{\hat{p}} f\right) \geq 1\right.$, dado que $D_{\hat{p}} f \cdot \hat{p}=0$ y entonces $\hat{p} \in \operatorname{ker}\left(D_{\hat{p}} f\right)$.) Por lo tanto $n=n-1+\operatorname{dim}\left(\operatorname{ker}\left(D_{\hat{p}} f\right)\right)$; es decir $\operatorname{dim}\left(\operatorname{ker}\left(D_{\hat{p}} f\right)\right)=1$, lo que implica que el rango de $D_{\hat{p}} f$ es $n-1$. (iv) $\Rightarrow$ (iii) por la prueba de la proposición 8 de [1]. (iii) $\Rightarrow$ (ii) por la prueba de la proposición 9 de [1]. (ii) $\Rightarrow$ (i) Como $C_{\hat{p}}=e, \hat{p}$ es un punto de equilibrio por definición.

Nota. La proposición anterior demuestra que el concepto de regularidad se reduce al concepto de regularidad definido en [1] para economías de intercambio.

Proposición 14 Sea $(f, A)$ una economía que satisface los supuestos del 1 al 7 . Entonces los puntos de equilibrio regular son puntos aislados en el conjunto de puntos de equilibrio de $(f, A)$.

Prueba. Sea $\hat{p}$ un punto de equilibrio regular de $(f, A)$. Considere la función $h=$ $g-i d_{X}$; sabemos por el teorema 9 que $h$ es diferenciable en un vecindario abierto $\sigma$ de $\hat{p}$. Entonces por el teorema de la función inversa $h$ es un difeomorfismo local alrededor de $\hat{p}$, en particular $h$ es una función inyectiva en un abierto $V \subseteq \sigma$ con $\hat{p} \in V$. Por lo tanto si $\hat{p}_{1}$ es un punto de equilibrio de $(f, A)$ tal que $\hat{p}_{1} \in V$ entonces $h\left(\hat{p}_{1}\right)=g\left(\hat{p}_{1}\right)-\hat{p}_{1}=0$; pero $h(\hat{p})=0$ luego $h\left(\hat{p}_{1}\right)=h(\hat{p})$ entonces $\hat{p}_{1}=\hat{p}$.

Por lo tanto $\hat{p}$ es un punto de equilibrio de $(f, A)$ aislado.

Teorema 15 Sea $(f, A)$ una economía que satisface los supuestos del 1 al 7 . Si $(f, A)$ es regular entonces el conjunto de puntos de equilibrio de $(f, A)$ es un conjunto finito.

Prueba. Si $h=g-i d_{X}$ sabemos que el conjunto de puntos de equilibrio de $(f, A)$ es $h^{-1}(0)$ (Teorema 3). Este conjunto es claramente un subconjunto cerrado de $X$. Por la proposición 14 todo punto de $h^{-1}(0)$ es aislado. Tenemos entonces que $h^{-1}(0)$ es un subconjunto cerrado y discreto del conjunto compacto $X$; por lo tanto $h^{-1}(0)$ debe ser un subconjunto finito de $X$.

En lo que resta del artículo estaremos interesados en probar un teorema del índice para economías $(f, A)$ regulares.

Lema 16 Sea $g: X \longrightarrow \mathbb{R}^{n}$ una función continua tal que $X \subseteq \mathbb{R}^{n}, X$ compacto. Entonces para todo $\varepsilon>0$ existe $h: X \longrightarrow \mathbb{R}^{n}$ función $C^{\infty}$ tal que $\|h(x)-g(x)\| \leq \varepsilon$ para todo $x \in X$.

Prueba. Por el teorema de Stone-Weierstrass existe una función polimonial en nvariables $h_{i}: X \longrightarrow \mathbb{R}$ tal que $\left|h_{i}(x)-g_{i}(x)\right| \leq \frac{\varepsilon}{\sqrt[2]{n}}$ para todo $x \in X$ y para todo $i=1, \cdots, n$; donde $g=\left(g_{1}, \cdots, g_{n}\right)$. Sea $h=\left(h_{1} \cdots, h_{n}\right)$, como $h_{i}$ es $C^{\infty}$ para todo $i$ entonces $h$ es $C^{\infty}$ y además tenemos que:

$$
(\|g(x)-h(x)\|)^{2}=\left(g_{1}(x)-h_{1}(x)\right)^{2}+\cdots+\left(g_{n}(x)-h_{n}(x)\right)^{2} \leq \frac{\varepsilon^{2}}{n^{2}}+\cdots+\frac{\varepsilon^{2}}{n^{2}}
$$

para todo $x \in X$ y entonces $\|g(x)-h(x)\| \leq \varepsilon$ para todo $x \in X$. 
Proposición 17 Sea $(f, A)$ una economía que satisface los supuestos del 1 al 7. Si $g: X \longrightarrow X$ tiene un conjunto finito de puntos fijos, entonces para todo $\epsilon>0$ existe una función $g^{*}: X \longrightarrow X$ de clase $C^{1}$ y un abierto $V$ de $\mathbb{R}^{n}$ tal que el conjunto de puntos fijos de $g$ está contenido en $V$ y tal que

(i) $\left\|g^{*}(x)-g(x)\right\| \leq \epsilon$ para todo $x \in X$.

(ii) $g^{*}(x)=g(x)$ para todo $x \in V \cap X$.

(iii) $g^{*}(x)=x$ implica que $x \in V$.

Prueba. Sea $\left\{p_{1}, \cdots, p_{k}\right\}$ el conjunto de puntos fijos de $g$. Sabemos del teorema 9 que para cada $p_{i}$ existe una bola $U_{i}$ de radio $\alpha_{i}$ y centro $p_{i}$ con $U_{i} \subseteq X$ y tal que $g$ es de clase $C^{1}$ en $U_{i}$.

Podemos escoger $\alpha_{1}, \cdots, \alpha_{k}$ tal que $U_{i} \cap U_{j}=\emptyset$ para todo $i, j \in\{1, \cdots, k\}, i \neq j$. Sean $\beta_{i}$ tal que $0<\beta_{i}<\alpha_{i}, V_{i}$ la bola abierta con centro $p_{i}$ y radio $\beta_{i}$ para $i=1, \cdots, k$, $V=\cup_{i=1}^{k} V_{i}$ y $U=\cup_{i=1}^{k} U_{i}$.

Tenemos que $g$ es de clase $C^{1}$ en $U$ y $V$ contiene los puntos fijos de $g$.

Para $i=1, \cdots, k$ sea $\vartheta_{i}: \mathbb{R}^{n} \longrightarrow \mathbb{R}$ una función de clase $C^{\infty}$ tal que $\vartheta_{i}(x)=1$ si $\left\|x-p_{i}\right\| \leq \beta_{i}, \vartheta_{i}(x)=0$ si $\left\|x-p_{i}\right\| \geq \alpha_{i}$ y $0<\vartheta_{i}(x)<1$ si $\beta_{i}<\left\|x-p_{i}\right\|<\alpha_{i}$.

Defina $\vartheta: \mathbb{R}^{n} \longrightarrow \mathbb{R}$ tal que $\vartheta(x)=\sum_{i=1}^{k} \vartheta_{i}(x)$. Como $\bar{V}_{i} \subseteq U_{i}$ para $i=1, \cdots, k$ entonces $\bar{V}_{i} \cap \bar{V}_{j}=\emptyset$ si $i \neq j$. Por otro lado si $x \in \bar{V}=\cup_{i=1}^{k} \bar{V}_{k}$ entonces existe $i$ tal que $x \in \bar{V}_{i}$, lo que implica que $x \in U_{i}$ y entonces $x \notin U_{j}$ para todo $j \neq i$ y así $\vartheta_{j}(x)=0$ para todo $j \neq i$; por lo tanto $\vartheta(x)=\vartheta_{i}(x)=1$. Entonces $\vartheta \equiv 1$ en $\bar{V}$.

Por otro lado si $p \in X \backslash U$ entonces $p \notin U_{i}$ y así $\vartheta_{i}(p)=0$ para todo $i$ por lo tanto $\vartheta(p)=\sum_{i=0}^{k} \vartheta_{i}(p)=0$. Se tiene que $\vartheta \equiv 0$ en $X \backslash U$. Si $p \in U \backslash \bar{V}$ existe $i \in\{1, \cdots, k\}$ tal que $p \in U_{i}$ y $p \notin \bar{V}_{j}$ para todo $j=1, \cdots, k$. Como $p \in U_{i}$ tenemos $p \notin U_{j}$ si $j \neq i$ y $p \in U_{i} \backslash \bar{V}_{i}$. Entonces $0<\vartheta_{i}(p)<1$ y $\vartheta_{j}(p)=0$ si $j \neq i$, luego $\vartheta(p)=\vartheta_{i}(p)$ y obtenemos que $0<\vartheta(p)<1$.

Hemos probado que $0<\vartheta<1$ en $U \backslash \bar{V}$.

Sabemos que $g(X) \subseteq S_{A}$ y que $S_{A}$ está contenido en el interior de $X$. Entonces tenemos que para todo $y \in g(X)$ existe $\delta_{y}>0$ tal que $B\left(y, 2 \delta_{y}\right) \subseteq$ interior de $X$.

La familia $\left\{B\left(y, 2 \delta_{y}\right) / y \in g(X)\right\}$ es un cubrimiento de $g(X)$ conjunto compacto, entonces existe $y_{1}, \cdots, y_{m}$ en $g(X)$ tal que $g(X) \subseteq \cup_{i=1}^{m} B\left(y_{i}, 2 \delta_{y_{i}}\right)$. La función $\|g(p)-p\|$ es mayor que 0 en $X \backslash V$ conjunto compacto, por lo tanto esta función alcanza su mínimo $\delta>0$ en $X \backslash V$. Sea $\epsilon>0$ tal que $0<\epsilon<\operatorname{mín}\left\{\delta_{y_{1}}, \cdots, \delta_{y_{m}}, \delta\right\}$, entonces por el lema anterior existe una función $h: X \longrightarrow \mathbb{R}^{n}$ de clase $C^{\infty}$ tal que $\|h(p)-g(p)\| \leq \epsilon$ para todo $p \in X$.

Probaremos que $h(X) \subseteq X$. Sea $p \in X$ se tiene que $\|h(p)-g(p)\| \leq \epsilon$, como $g(p) \in X$ existe, $i \in\{1, \cdots, m\}$ tal que $g(p) \in B\left(y_{i}, \delta_{y_{i}}\right)$ es decir $\left\|g(p)-y_{i}\right\|<\delta_{y_{i}}$ y entonces $\left\|h(p)-y_{i}\right\| \leq\|h(p)-g(p)\|+\left\|g(p)-y_{i}\right\|<\epsilon+\delta_{y_{i}}<2 \delta_{i}$.

Por lo tanto $h(p) \in B\left(y_{i}, 2 \delta_{i}\right) \subseteq$ interior $\operatorname{de} X$; luego $h(p) \in X$ para todo $p \in X$, es decir $h(X) \subset X$. Tenemos entonces una función $h: X \longrightarrow X$ de clase $C^{\infty}$ tal que $\|h(p)-g(p)\| \leq \epsilon$ para todo $x \in X$.

Defina $g^{*}: X \longrightarrow X$ tal que $g^{*}(p)=\vartheta(p) g(p)+(1-\vartheta(p)) h(p)$.

Hemos visto que existe $y_{i} \in g(X)$ tal que $g(p) \in B\left(y_{i}, \delta_{i}\right), h(p) \in B\left(y_{i}, 2 \delta_{i}\right)$ y $B\left(y_{i}, 2 \delta_{i}\right) \subseteq$ en el interior de $X$. Por lo tanto $h(p), g(p) \in B\left(y_{i}, 2 \delta_{i}\right)$ y como $B\left(y_{i}, 2 \delta_{i}\right)$ es convexo se tiene que $g^{*}(p) \in B\left(y_{i}, 2 \delta_{i}\right)$ y entonces $g^{*}(p) \in X$ para todo $p \in X$. Como 
$g, h, \vartheta$ son de clase $C^{1}$ en $X$ entonces $g^{*}$ es de clase $C^{1}$ en $X$. Si $p \in V$ sabemos que $\vartheta(p)=1$ por lo tanto $g^{*}(p)=g(p)$, en particular $g\left|V=g^{*}\right| V$. Sea $p \in X$ se tiene que:

$\left\|g^{*}(p)-g(p)\right\|=\|(1-\vartheta(p))(h(p)-g(p))\|=(1-\vartheta(p))\|h(p)-g(p)\| \leq\|h(p)-g(p)\| \leq \epsilon$.

Entonces se tiene que $\left\|g^{*}(p)-g^{*}(p)\right\| \leq \epsilon$ para todo $p \in X$.

Sea $\hat{p} \in X$ punto fijo de $g^{*}$. Suponga que $\hat{p} \in X \backslash V$ entonces $\|g(\hat{p})-\hat{p}\| \geq \delta$. Como $\left\|g^{*}(\hat{p})-g(\hat{p})\right\| \leq \epsilon$, tenemos que $\|g(\hat{p})-\hat{p}\| \leq \epsilon$ y entonces $\delta \leq \epsilon$; pero $\epsilon<\delta$, lo cual es una contradicción y entonces $\hat{p} \in V$. Hemos demostrado que $g^{*}$ tiene las propiedades requeridas.

Sea $X$ una $n$-variedad topológica orientable con frontera; para cada función $g: X \longrightarrow$ $X$ se define el número de Lefschetz $L(g) \in \mathbb{Z}$ de $g$ ver [4]. Cuando $X$ es una n-variedad con frontera $C^{1}$ y $g: X \longrightarrow X$ una función de clase $C^{1}$ con un número finito de puntos fijos, tal que para todo punto fijo $\hat{p} \operatorname{de} g$ se tiene $\operatorname{det}\left[D_{\hat{p}} g-I_{n}\right] \neq 0$. Entonces $L(g)=\sum_{g(\hat{p})=\hat{p}} \operatorname{sig}\left(\operatorname{det}\left(D_{\hat{p}} g-I_{n}\right)\right)$. (ver por ejemplo [4],[2],[3]). El teorema del punto fijo de Lefschetz dice que si $L(g) \neq 0$ entonces $g$ tiene al menos un punto fijo (ver [4]). Aplicaremos esta teoría del punto fijo de Lefschetz a nuestras funciónes $g, g^{*}: X \longrightarrow X$.

Teorema 18 Sea $(f, A)$ una economía que satisface los supuestos del 1 al 5 . Entonces $L(g)=(-1)^{n}$, en particular $(f, A)$ posee al menos un punto de equilibrio.

Prueba. Como $X$ puede ser escogido como una n-bola cerrada, podemos suponer que $X$ es convexo. Sea $S: X \longrightarrow X$ tal que $S(x)=\left(\frac{1}{n}, \cdots, \frac{1}{n}\right)$ para todo $x \in X$ y considere la homotopía $G: X \times[0,1] \longrightarrow X$ tal que $H(p, t)=(1-t) S(p)+t g(p)$.

Tenemos que $H(p, t) \in X$ dado $X$ es convexo. Entonces las funciones $S$ y $g$ son homotópicas y por lo tanto $L(S)=L(g)$. S sólo tiene un punto fijo $\hat{p_{0}}=\left(\frac{1}{n}, \cdots, \frac{1}{n}\right)$ y luego $L(S)=$ $\operatorname{sig}\left(\operatorname{det}\left(D_{\hat{p}_{0}} S-I_{n}\right)\right)=\operatorname{sig}\left(\operatorname{det}\left(-I_{n}\right)\right)=\operatorname{sig}(-1)^{n}=(-1)^{n}$.

Por lo tanto $L(g)=(-1)^{n}$. Por el teorema del punto fijo de Lefschetz como $L(g) \neq 0$ existe $\hat{p} \in X$ tal que $g(\hat{p})=\hat{p}$; es decir $\hat{p}$ es un punto de equilibrio de $(f, A)$.

Corolario 19 Sea $(f, A)$ una economía que satisface los supuestos del 1 al 7. Si $g: X \longrightarrow X$ tiene un conjunto finito de puntos fijos. Entonces $L\left(g^{*}\right)=(-1)^{n}$.

Prueba. Es suficiente demostrar que $g$ y $g^{*}$ son homotópicas. Como $X$ es convexo entonces si $t \in[0,1]$ y $x \in X$, tenemos que $t g(x)+(1-t) g^{*}(x) \in X$ por lo tanto $H:[0,1] \times$ $X \longrightarrow X$ tal que $H(t, x)=\operatorname{tg}(x)+(1-t) g^{*}(x)$ es una homotopía. Entonces se tiene que $L\left(g^{*}\right)=L(g)=(-1)^{n}$.

Definición 7 Sea $(f, A)$ una economía que satisface los supuestos del 1 al 7 y $\hat{p}$ un punto de equilibrio de $(f, A)$ regular. El indice de $\hat{p}$ se define como sig $\left(\operatorname{det}\left[\begin{array}{ll}-D_{\hat{p}} f & C_{\hat{p}} \\ -C_{\hat{p}}^{t} & 0\end{array}\right]\right)$ y se denota por ind $(\hat{p})$.

Proposición 20 Sea $\left(f,-I_{n}\right)$ una economía de intercambio que satisface los supuestos 1,2 y 3 y $\hat{p}$ un punto de equilibrio regular de $\left(f,-I_{n}\right)$ tal que $p_{i}>0$ para todo $i$. Entonces ind $(\hat{p})=\operatorname{sig}\left(\operatorname{det}\left[\begin{array}{ll}-D_{\hat{p}} f & \hat{p} \\ -\hat{p}^{t} & 0\end{array}\right]\right)$. 
Prueba. $\quad$ La proposición 9 de [1], afirma que el signo de $\operatorname{det}\left[\begin{array}{cc}D_{\hat{p}} f & q \\ q^{t} & 0\end{array}\right]$ no depende de $q$ si $q \in \mathbb{R}^{n} \backslash\{0\}$. En particular tenemos que $\operatorname{sig}\left(\operatorname{det}\left[\begin{array}{cc}D_{\hat{p}} f & \hat{p} \\ \hat{p}^{t} & 0\end{array}\right]\right)=\operatorname{sig}\left(\operatorname{det}\left[\begin{array}{cc}D_{\hat{p}} f & e \\ e^{t} & 0\end{array}\right]\right)$. Multiplicando esta ecuación por $(-1)^{n}$ tenemos que

$$
\operatorname{sig}\left(\operatorname{det}\left[\begin{array}{ll}
-D_{\hat{p}} f & \hat{p} \\
-\hat{p}^{t} & 0
\end{array}\right]\right)=\operatorname{sig}\left(\operatorname{det}\left[\begin{array}{ll}
-D_{\hat{p}} f & e \\
-e^{t} & 0
\end{array}\right]\right),
$$

pero como $C_{\hat{p}}=e$ entonces $\operatorname{sig}\left(\operatorname{det}\left[\begin{array}{ll}-D_{\hat{p}} f & \hat{p} \\ -\hat{p}^{t} & 0\end{array}\right]\right)=\operatorname{sig}\left(\operatorname{det}\left[\begin{array}{ll}-D_{\hat{p}} f & e \\ -C_{\hat{p}}^{t} & 0\end{array}\right]\right)$. es decir $\operatorname{ind}(\hat{p})=\operatorname{sig}\left(\operatorname{det}\left[\begin{array}{ll}-D_{\hat{p}} f & \hat{p} \\ -\hat{p}^{t} & 0\end{array}\right]\right)$

Nota. La proposición anterior demuestra que el concepto de indice dado en la definición anterior se reduce al concepto de índice estudiado para economías de intercambio en [1].

Proposición 21 Sea $(f, A)$ una economía que satisface los supuestos del 1 al 7 y $\hat{p}$ un punto de equilibrio regular. Entonces ind $(\hat{p})=(-1)^{n} \operatorname{sig}\left(\operatorname{det}\left(D_{\hat{p}} g-I_{n}\right)\right)$.

Prueba. De la proposición 11 sabemos que $\operatorname{sig}\left(\operatorname{det}\left(D_{\hat{p}} g-I_{n}\right)\right)=\operatorname{sig}\left(\operatorname{det}\left[\begin{array}{ll}D_{\hat{p}} f & C_{\hat{p}} \\ C_{\hat{p}}^{t} & 0\end{array}\right]\right)$ entonces

$$
\begin{aligned}
(-1)^{n} \operatorname{sig}\left(\operatorname{det}\left(D_{\hat{p}} g-I_{n}\right)\right) & =(-1)^{n} \operatorname{sig}\left(\operatorname{det}\left[\begin{array}{ll}
D_{\hat{p}} f & C_{\hat{p}} \\
C_{\hat{p}}^{t} & 0
\end{array}\right]\right)=\operatorname{sig}\left((-1)^{n} \operatorname{det}\left[\begin{array}{ll}
D_{\hat{p}} f & C_{\hat{p}} \\
C_{\hat{p}}^{t} & 0
\end{array}\right]\right) \\
& =\operatorname{sig}\left(\operatorname{det}\left[\begin{array}{ll}
-D_{\hat{p}} f & C_{\hat{p}} \\
-C_{\hat{p}}^{t} & 0
\end{array}\right]\right)=\operatorname{ind}(\hat{p}) .
\end{aligned}
$$

Teorema 22 Sea $(f, A)$ una economía regular que satisface los supuestos del 1 al $\%$. Entonces $\sum_{\hat{p} \in \Pi(f, A)}$ ind $(\hat{p})=1$.

Prueba. Sabemos del teorema 15 que $\Pi(f, A)$ es un conjunto finito y del teorema 18 que $\Pi(f, A) \neq \emptyset$.

Entonces tenemos que:

$$
\begin{aligned}
\sum_{\hat{p} \in \Pi(f, A)} \operatorname{ind}(\hat{p}) & =(-1)^{n} \sum_{g(\hat{p})=\hat{p}} \operatorname{sig}\left(D_{\hat{p}} g-I_{n}\right) \\
& =(-1)^{n} \sum_{g^{*}(\hat{p})=\hat{p}} \operatorname{sig}\left(D_{\hat{p}} g^{*}-I_{n}\right) \\
& =(-1)^{n} L\left(g^{*}\right) \\
& =(-1)^{n} \cdot(-1)^{n}=1
\end{aligned}
$$

El teorema anterior es conocido como el teorema del índice. Como el concepto de índice para puntos de equilibrio regulares se reduce al concepto correspondiente estudiado en [1] para economías de intercambio, el teorema 22 es una generalización del teorema del índice probado en [1] para economías de intercambio.

Agradezco las sugerencias y observaciones dadas por el Dr. Fernán Ulate Montero, las cuales fueron muy útiles en la realización de este trabajo. 
O. ACUÑA

\section{Referencias}

[1] Acuña Ortega, O.; Ulate Montero, F. (1994) "Equilibrio Walrasiano en economías de intercambio", Revista de Matemáticas: Teoría y Aplicaciones 1(1): 1-6.

[2] Bott, R.; Atiyah, M. (1966) "A Lefschetz fixed point formula for elliptic differential operators", Bull. Amer Math. Soc. 72: 245-250.

[3] Brown, R.F. (1965) "On the Lefschetz fixed point theorem", Amer. J. Math. 87: 1-10. (1965).

[4] Greenberg, J.M. (1974) Lecture on Algebraic Topology. W. A. Benjamin, Inc., Advanced Book Program.

[5] Guillermin, V.; Pollack, A. (1974) Differential Topology. Prentice-Hall, Englewood Cliffs.

[6] Kehoe, T. (1980) "An index theorem for general equilibrium models with productions", Econometrica 48(5): 1211-1232.

[7] Koopmans, T. (1951) "Analysis of production as an efficient combination of activities", in: T. Koopmans (Ed.) Activity Analysis of Production and Allocation, John Wiley \& Sons, New York: 33-97.

[8] Mas-Colell, A. (1985) The Theory of General Economic Equilibrium. A Differentiable Approach. Cambridge University Press, Cambridge. 